Cite this: RSC Advances, 2013, 3, 11846

Received 29th January 2013, Accepted 6th May 2013

DOI: $10.1039 / c 3 r a 40491 a$

www.rsc.org/advances

\section{Immuno-CoPS (conducting paper strips) for futuristic cost-effective cancer diagnostics}

\author{
Saurabh Kumar, tab $^{\text {ab }}$ shore Kumar Jagadeesan, $+^{\text {tac }}$ Amish G. Joshi ${ }^{\mathrm{a}}$ \\ and Gajjala Sumana*a
}

\begin{abstract}
The fabrication of cost-effective, rapid and specific paper based point-of-care (POC) diagnostics based on the principles of bio-recognition is currently at the vanguard of biomedical research, as it is playing a key role in early diagnosis and treatment, resulting in the inhibition of infection and reducing the mortality rate. In the present report, we demonstrate that biomolecules can be directly attached to a paper matrix covalently, by suitable modification of the paper matrices. A high conductivity is achieved and the porosity can be tailored and has been utilized for the quantitative estimation of bio-analytes. We prove the feasibility of our approach for the detection of cancer biomarkers in a wide detection range.
\end{abstract}

\section{Introduction}

For advanced developments in POC devices, the selection of the matrix and the immobilization of bio-analytes for the rapid and sensitive detection of bio-recognition through suitable processes to obtain elevated signals is a major challenge. Utilizing the principles of nanotechnology and biotechnology, presently a wide range of materials have been employed as the matrices for biosensing. ${ }^{1-6}$ Among them, paper-based POC diagnostics have enormous potential as next generation biosensors, because of their prospects for the design of lowcost, portable and user friendly analytical devices, with the obvious advantages of safe disposal and biosafety management. Paper-based strips have recently been explored for the qualitative analysis of various bio-analytes. Recently, Whitesides and his group have introduced the miniaturized "POCKET Immunosensor" based on paper-based substrates. ${ }^{7}$ A new POC system combining a microfluidic paper based analytical device and a handheld optical colorimeter has also been developed for finding the concentration of specific analytes in biological fluids. In the recent past, a new inexpensive method for patterning paper with wax was introduced by $\mathrm{Lu}$ et $a .^{8}{ }^{8}$ As an alternative to plastic 96 well plates, a new type of paper-based microarray was created using a photolithography technique. ${ }^{9}$ This multi-zone paper plate enables the parallel and multiplexed analysis of samples. A

${ }^{a}$ CSIR- National Physical Laboratory, Dr K. S. Krishnan Road, New Delhi, 110012, India. E-mail: sumanagajjala@gmail.com; Fax: +91 11 45609310; Tel: +91 11 45609152

${ }^{b}$ Department of Biotechnology, Delhi Technological University, Shahbad Daulatpur, Delhi 110042, India

${ }^{c}$ Dept. of Measurement Technology and Industrial Electrical Engineering, Lund University, SE-221 00, Lund, Sweden

$\dagger$ Authors with equal contribution. non-conventional paper-based ELISA comprising the ASSURED criteria has been developed with a faster detection time. $^{10}$

Many efforts have been directed toward developments in fabrication and functionalization of quantitative methods for paper-based devices, to enhance the sensitivity of the detection and stability of the POC biosensing strips. The performance of the biosensor can be improved through the surface functionalization of the paper with suitable functional groups that enable a proper interaction with biomolecules; which ultimately lead to quantitative detection. The surface modification of paper with multi-walled carbon nanotubes and chitosan for the development of electrochemical immunosensors was reported by Zang et al. ${ }^{11}$ They reported a high-throughput, smart, 3D microfluidic paper-based device, utilized for the detection of the cancer biomarker r-fetoprotein (AFP), carcinoma antigens CA-125 and CA-199, and carcinoembryonic antigen (CEA) having detection limits of $0.01 \mathrm{ng} \mathrm{mL} \mathrm{g}^{-1}, 6.0 \mathrm{mU}$ $\mathrm{mL}^{-1}, 8.0 \mathrm{mU} \mathrm{mL}{ }^{-1}$ and $5.0 \mathrm{pg} \mathrm{mL}^{-1}$, respectively. However, wax-printing followed by screen-printing and chemical treatment, make this device preparation intricate and expensive. The modification of paper with graphene and gold nanoparticles for the sensitive and simultaneous diagnosis of a panel of breast cancer markers, CA-153, CA-125 and CEA, with limits of detection $1.5 \times 10^{-3} \mathrm{U} \mathrm{mL}^{-1}, 3.4 \times 10^{-4} \mathrm{U} \mathrm{mL}^{-1}$, and 1.2 $\times 10^{-3} \mathrm{ng} \mathrm{mL}^{-1}$, respectively has also been reported. ${ }^{12}$ Many analytical methods including immunoradiometric assay, enzyme-linked immunosorbent assay, indirect fluorescent antibody techniques, immunocytochemical inhibition assay and an immunohistochemical technique have been employed in the detection of the soluble interleukin-2 receptor alpha, sIL2R $\alpha .{ }^{13-17}$ Recently, electrochemical immunosensors with a high orientation of electrode-bound antibodies have shown promise as a successful technique for the detection of a wide 
range of analytes with an enhanced stability. ${ }^{18,19}$ However, the appropriate tailoring of a suitable substrate for the better immobilization of antibodies for the desired sensitivity and stability is extremely vital for the fabrication of an electrochemical immunosensor for sIL2R $\alpha$ detection.

Conducting polymers are multifunctional materials that can be employed as receptors as well as transducers or immobilization matrices in electrochemical biosensing. These are attractive alternative methods involving the formation of a biomolecule film from biological entities functionalized by electro-polymerizable groups, or the covalent binding of the biomolecule to polymer films bearing appropriate functional groups. Recently, a wide number of applications have been explored using the modification of paper with these conducting polymers. ${ }^{20-22}$ These printing and coating techniques are promising for the paper modification, and electrochemical deposition procedures allow the reproducible and precise formation of a polymer-coated surface. Electrochemical polymerization thus constitutes an attractive method for the preparation of biochips. In the present work, pre-treated paper electrodes using a screen printing technique were chemically modified by polyaniline deposition through an electrochemical polymerization, to obtain amino functional pendant groups, which are suitable for the grafting of antibodies. Conducting paper strips (CoPS) and antibody immobilized conducting paper strips (Immuno-CoPS) have been characterized using detailed electrochemical, morphological and spectroscopic investigations. The conductivity of the polymer coating is studied exclusively as a function of the number of scans and the detailed structure property correlations have also been elucidated.

\section{Experimental section}

\section{Materials and chemicals}

Whatman filter paper (Grade 1, with a pore size of $11 \mu \mathrm{m}$ ) was used for fabricating the conducting paper electrodes. Aniline was purchased from Merck India Limited and was distilled before use. Graphite and silver powders were purchased from BHEL, India. The monoclonal antibody of sIL2R $\alpha$ and Human $\operatorname{sIL} 2 \mathrm{R} \alpha$ were purchased from Sigma-Aldrich, India. All the biochemicals, chemicals and reagents were of analytical grade and were used without further purification.

\section{Fabrication of immuno-conducting paper strips (Immuno- CoPS)}

The strategy used for the fabrication of label free immunosensors for cancer detection, using nanostructured polyaniline-paper strips is depicted in Scheme 1. Initially, a silver pattern followed by carbon patterns were screen-printed on the surface of the filter paper. Then, they were allowed to dry for 20-30 min. Nanostructured polyaniline (PANI) was deposited onto the filter paper by polymerization of the aniline in an acidic medium $(\mathrm{pH}=1)$ by adopting a cyclic voltammetric technique at a potential cycling $(n=5,10,15,20)$ between
-200 and $+1200 \mathrm{mV}$ versus a $\mathrm{Ag} / \mathrm{AgCl}$ reference electrode at a scan rate of $100 \mathrm{mV} \mathrm{s}^{-1}$.

The covalent immobilization of anti-sIL2R $\alpha$ on the surface of the CoPS was done using 1-ethyl-3-[3-dimethylaminopropyl]carbodiimide hydrochloride as a cross-linker in the presence of $\mathrm{N}$-hydroxysuccinimide (EDC-NHS). For this purpose, the CoPS electrode was incubated at $4{ }^{\circ} \mathrm{C}$ for about $6 \mathrm{~h}$ in a solution of $0.2 \mu \mathrm{g} \mathrm{ml}{ }^{-1}$ of anti-sIL2R $\alpha$ containing $0.2 \mathrm{M}$ EDC and 0.1 M NHS. The electrode was then rinsed with phosphate buffer (PBS, $50 \mathrm{mM}, \mathrm{pH} 7.2,0.9 \% \mathrm{NaCl}$ ) to remove unbound antibodies, and $1 \%$ of bovine serum albumin (BSA) in PBS was added to the antibody-modified electrode to block unspecific sites. After rinsing again with PBS, the electrodes were stored at $4{ }^{\circ} \mathrm{C}$ before carrying out the sensing studies.

\section{Measurements and characterization}

The electrochemical characterization and electrochemical polymerization were performed using an Autolab potentiostat/galvanostat (Autolab, Netherlands). All the electrochemical measurements were carried out using a three-electrode cell with CoPS as the working electrode, platinum as the auxiliary electrode and a $\mathrm{Ag} / \mathrm{AgCl}$ electrode as the reference electrode in phosphate buffer (PBS, $50 \mathrm{mM}, \mathrm{pH} 7.2,0.9 \% \mathrm{NaCl}$ ) containing $5 \mathrm{mM}\left[\mathrm{Fe}(\mathrm{CN})_{6}\right]^{3-/ 4-}$. Fourier transform infrared (FTIR) spectra of CoPS and anti-sIL2R $\alpha /$ CoPS were recorded using a Perkin-Elmer, Spectrum BXII spectrometer in transmission mode in the region of $400-4000 \mathrm{~cm}^{-1}$. The morphology of the bio-electrodes was investigated using a scanning electron microscope (LEO 440).

X-ray photoelectron spectroscopic (XPS) investigations were carried out using a Perkin Elmer 1257 model, operating at a base pressure of $\geqslant 3.2 \times 10^{-8}$ Torr at $300 \mathrm{~K}$ with a non-monochromatized $\mathrm{Mg}-\mathrm{K} \alpha$ line at $1253.6 \mathrm{eV}$. The overall instrumental resolution was about $\geqslant 300 \mathrm{meV}$. Pass energies for a general survey scan and the core level spectra were kept at 143.05 and $71.55 \mathrm{eV}$ with measurement steps of 0.5 and $0.05 \mathrm{eV}$, respectively.

\section{Results and discussion}

The nanostructured polyaniline deposition onto filter papers was successfully demonstrated by morphological investigations using scanning electron microscopy (SEM) (Fig. 1A). The CoPS show a well aligned porous nanofibrous/nanostructured three-dimensional inter-connected network morphology. Whereas, after the immobilization of anti-sIL2R $\alpha$ (Fig. 1B), small globules representing the antibody at the chain ends of the polyaniline matrix, indicate the presence of biomolecules on the surface. The electrode exhibits a smooth nanofibrous structure and a shiny appearance due to the accumulation of static charge, revealing the successful covalent immobilization of the antibody. Electron diffraction X-ray analysis of the CoPS electrode (Fig. 2A) shows the existence of nitrogen, which confirms the presence of the amino group of aniline, whereas the presence of oxygen in the Immuno-CoPS (Fig. 2B) confirms antibody immobilization. 


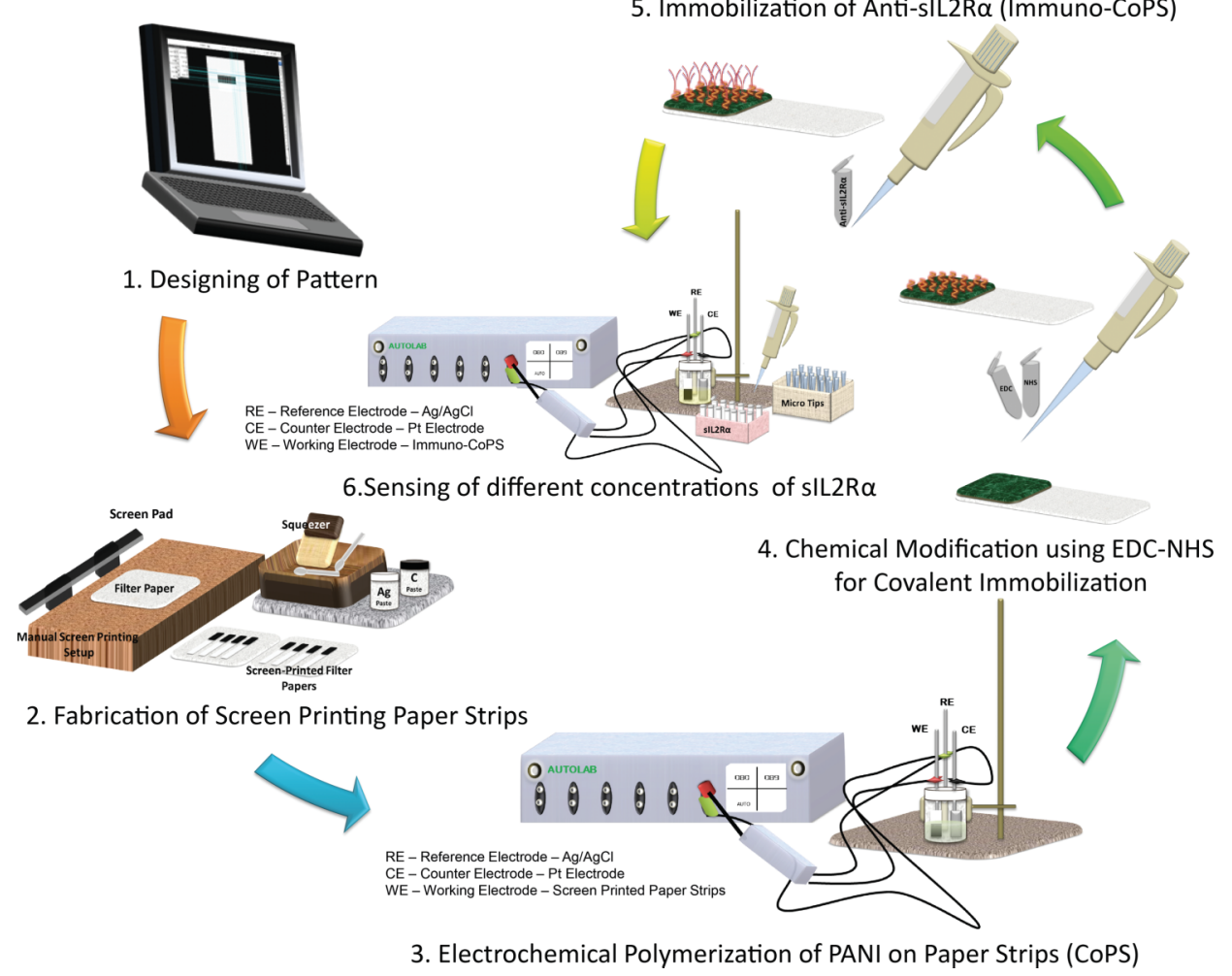

5. Immobilization of Anti-sIL2Ra (Immuno-CoPS)

Scheme 1 Schematic representation of the fabrication of Immuno-CoPS and the detection of sIL2R $\alpha$ using Immuno-CoPS.

The presence of polyaniline in its conducting emeraldine base form on the surface of the conducting paper strips (CoPS) was confirmed by FTIR spectroscopic analysis (Fig. 2C (i)), by the benzenoid ring and quinoid ring peaks at $1421 \mathrm{~cm}^{-1}$ and $1585 \mathrm{~cm}^{-1}$, respectively. ${ }^{23}$ The emeraldine base dominancy is shown by the higher intensity of the quinoid peak compared to that of the benzenoid peak. ${ }^{24,25} \mathrm{C}=\mathrm{N}$ vibrations are confirmed by the peaks at $1590 \mathrm{~cm}^{-1}$. C-N stretching of the aromatic amine, in-plane $\mathrm{C}-\mathrm{H}$ bending of the quinoid ring and out-ofplane bending of the $\mathrm{C}-\mathrm{H}$ bond in the aromatic ring are confirmed by the peaks at $1269 \mathrm{~cm}^{-1}, 1176 \mathrm{~cm}^{-1}$ and 820 $\mathrm{cm}^{-1}$, respectively. ${ }^{26,27}$ Similarly, The FTIR (Fig. 2C (ii)) peak at $1680 \mathrm{~cm}^{-1}$ in the spectrum of the bio-electrode after the immobilization of the antibody anti-sIL2R $\alpha$, indicates the presence of the $\mathrm{C}=\mathrm{O}$ stretching for amide $\mathrm{I}$. The $\mathrm{C}-\mathrm{N}$ stretching and $\mathrm{N}-\mathrm{H}$ bending at $1550 \mathrm{~cm}^{-1}$ for amide $\mathrm{II}^{28,29}$ confirms the anti-sIL2R $\alpha$ immobilization onto the CoPS electrode.

Before carrying out a detailed XPS analysis, the conductivity measurements using a four-probe method, as a function of the number of potential cycles, as well as time of deposition were carried out and the results are depicted [Table 1]. It was found that the bare screen-printed paper electrode exhibits the conductivity at $199.85 \mathrm{~S} \mathrm{~cm}^{-2}$, whereas after polyaniline deposition over 5 cycles, the conductivity was enhanced significantly to $2849 \mathrm{~S} \mathrm{~cm}^{-2}$. However, after increasing the number of cycles $(n=10)$, the conductivity was found to fall radically. This is attributed to the increased thickness of the film. As expected, after 15 cycles, the conductivity further dropped to $1.23 \mathrm{~S} \mathrm{~cm}^{-2}$.

The chemical composition analysis of the PANI films at different scan cycles (5, 10, 15 and 20) was studied using XPS analysis. The XPS survey scan (Fig. 3) of the PANI samples shows the presence of $\mathrm{N}(1 \mathrm{~s})(\sim 400 \mathrm{eV}), \mathrm{C}(1 \mathrm{~s})(\sim 285 \mathrm{eV}), \mathrm{Cl}$ (2p) $(\sim 199 \mathrm{eV})$ and $\mathrm{O}(1 \mathrm{~s})(\sim 532 \mathrm{eV})$. All the spectra were corrected to the $\mathrm{C}(1 \mathrm{~s})$ peak $(285 \mathrm{eV})$. The elements carbon and nitrogen originate from the PANI backbone, whereas the element chlorine is a counter ion in the case of the protonated PANI samples. Oxygen, in the polyemeraldine base, originated from partial oxidation of the films' surface, or from weakly complexed oxygen atoms.

In particular, the proportions of the quinoid imine $(=\mathrm{N}-$ moiety), benzenoid amine (-NH- moiety), and the positively charged nitrogen, corresponding to a particular oxidation and protonation level of the polymer, can be quantitatively differentiated. Therefore, a systematic deconvolution was performed using peak-fit software with a linear background subtraction and providing reference binding energy values for species and full width at half maxima [Table 2] on $\mathrm{N}(1 \mathrm{~s})$ and $\mathrm{C}$ (1s) core level spectra, which are shown in Fig. 4 and Fig. 5, respectively. It is evident from Fig. 4 that $N(1 \mathrm{~s})$ has four species: N1-neutral imine nitrogen $(\sim 398.5 \mathrm{eV})$, N2-neutral amine nitrogen $(\sim 399.5 \mathrm{eV}), \mathbf{N 3}$-oxidized amine $(\sim 400.8 \mathrm{eV})$ and N4-protonated imine $(\sim 402.2 \mathrm{eV})$.

The systematic change in the deconvoluted species $\mathrm{N}(1 \mathrm{~s})$ was performed and the \% area under the curve for each 

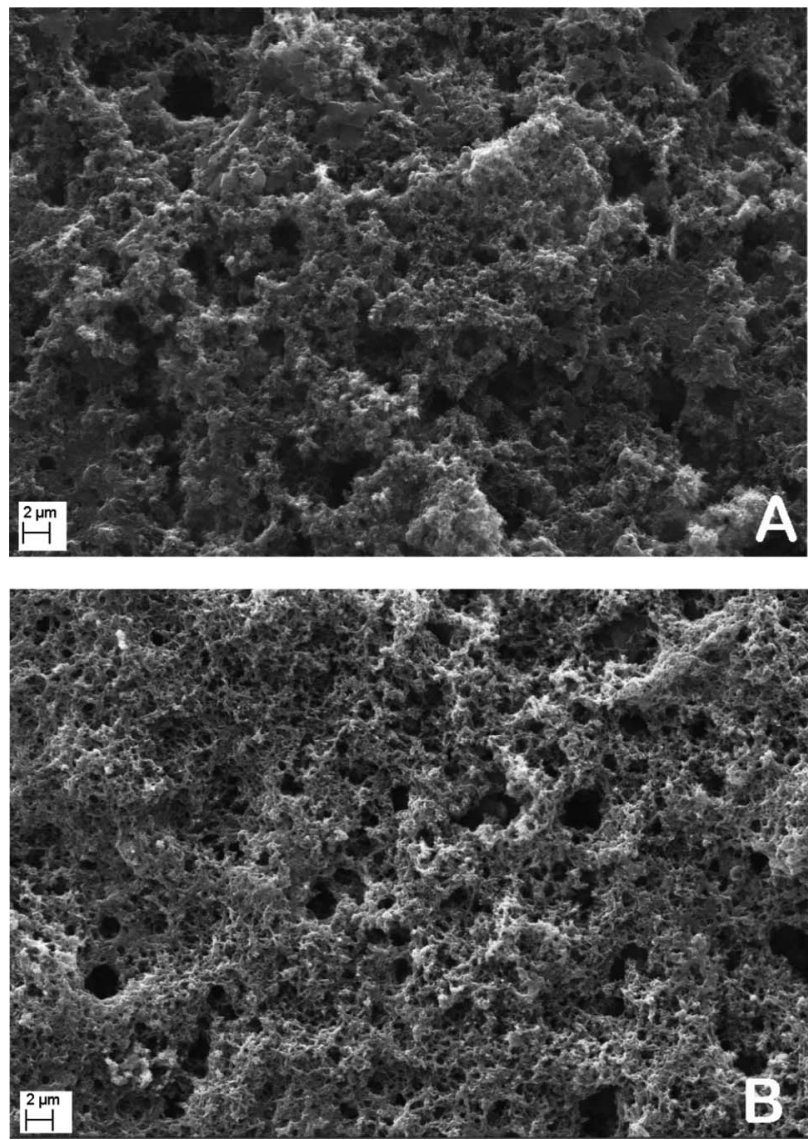

Fig. 1 Morphological investigations of (A) CoPS and (B) Immuno-CoPS using a scanning electron microscope at a magnification of 5000x.

species was obtained. The change in the \% area of the species, plotted as a function of the number of scans is shown in Fig. 6a. The decrease in the peak corresponds to $\mathbf{N 4}$ and the increase in the peak $\mathbf{N 1}$, with respect to the number of scans, shows the de-protonation of the protonated imine, resulting in the formation of a neutral imine, which is generally a barrier to charge transfer. This reflects the development of a more neutral imine nitrogen species, corresponding to the transformation of the conductive emeraldine form into the neutral and reduced leucoemeraldine form of polyaniline. ${ }^{30,31}$

The systematic deconvolution of the XPS C (1s) core level spectrum of PANI (Fig. 5) shows several different features, with binding energies spanning the range from $\sim 287 \mathrm{eV}$ to 284 $\mathrm{eV} .^{32}$ The spectrum contains a large peak centred at around $285 \mathrm{eV}$ and has a slight asymmetric broadening towards high binding energies, due to a combination of the protonation of imine and amine sites. The $\mathrm{C}(1 \mathrm{~s})$ core level spectra can be deconvoluted into four peaks: C1 - neutral C-C/C-H $(284.5 \mathrm{eV})$ bonds, C2 - neutral $\mathrm{C}-\mathrm{N} / \mathrm{C}=\mathrm{N}(285.4 \mathrm{eV})$ bonds, C3 $-\mathrm{C}^{-} \mathrm{N}^{+}$ $(286.4 \mathrm{eV})$ carbon atoms bonded to polaronic-type and $\mathbf{C 4}$ $\mathrm{C}=\mathrm{N}^{+}(287.4 \mathrm{eV})$ carbon atoms bonded to bipolaronic-type nitrogen atoms.

The depletion (Fig. 6b) of the protonated imine and the enhancement of neutral amine groups, which correspond to
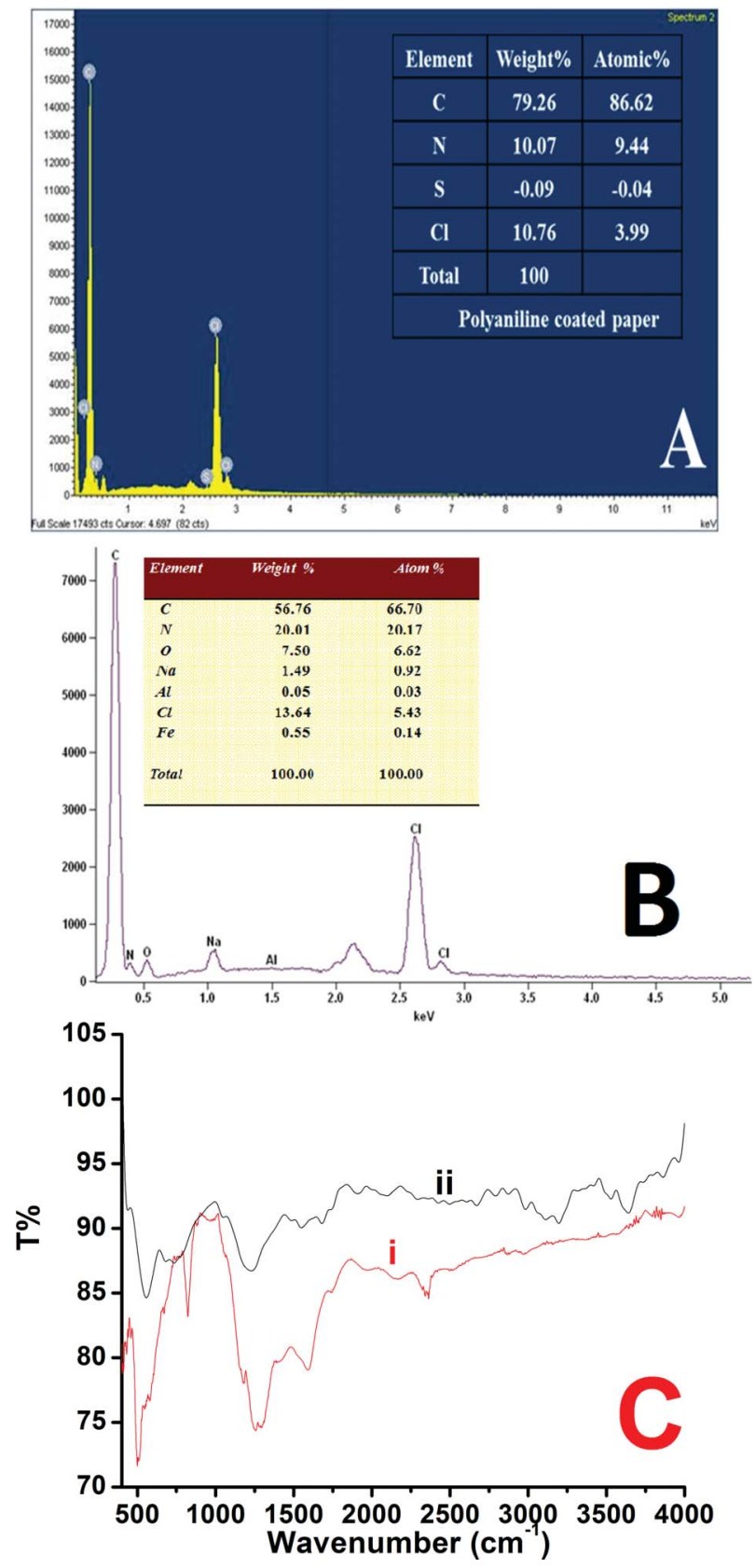

Fig. 2 Energy-dispersive X-ray spectroscopy of (A) conducting paper strips and (B) Immuno-CoPS. (C) FTIR spectra of (i) CoPS and (ii) Immuno-CoPS.

Table 1 Conductivity of polyaniline-paper strips as a function of the number of polymerization cycles

\begin{tabular}{lcc}
\hline No. of cycles & Deposition time $(\mathrm{s})$ & Conductivity $\left(\mathrm{S} \mathrm{cm}^{-2}\right)$ \\
\hline $\mathbf{0}$ (bare electrode) & 0 & 199.85 \\
$\mathbf{5}$ & 199 & 2849.00 \\
$\mathbf{1 0}$ & 398 & 328.96 \\
$\mathbf{1 5}$ & 597 & 1.23
\end{tabular}




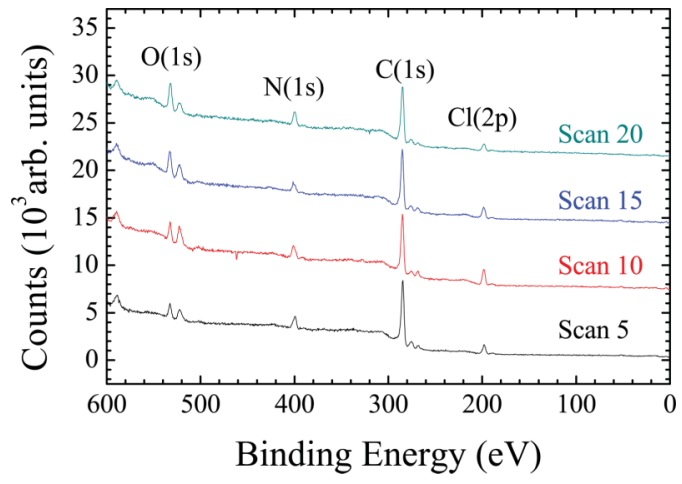

Fig. 3 XPS survey scan spectra for polyaniline as a function of number of polymerization cycles of polyaniline.

the formation of the reduced leucoemeraldine state, are confirmed with the descending and ascending trends of $\mathbf{C 4}$ and $\mathbf{C 1}$, respectively. Hence, from following the trends of the species $\mathbf{N 1}, \mathbf{N 4}, \mathbf{C} \mathbf{1}$ and $\mathbf{C 4}$, it is clear that the increase in the number of scan cycles leads to the transformation of the conducting emeraldine state into the neutral leucoemeraldine state of polyaniline, on the surface of the paper electrode.

Cyclic voltammetry (CV) was performed on the BSA/antisIL2R $\alpha /$ CoPS bio-electrode in a phosphate buffer (PBS, $50 \mathrm{mM}$, $\mathrm{pH} 7.2,0.9 \% \mathrm{NaCl})$, containing $5 \mathrm{mM}\left[\mathrm{Fe}(\mathrm{CN})_{6}\right]^{3-14-}$, as a function of the scan rate from $10-90 \mathrm{mV} \mathrm{s}^{-1}$ (Fig. 7). It can be seen that the magnitude of both anodic $\left(I_{\mathrm{a}}\right)$ and cathodic $\left(I_{\mathrm{c}}\right)$ current exhibits a linear relationship with the square root of the scan rate (Fig. 7A), revealing a diffusion-controlled process. Also, the peak to peak separation potential $\left[\Delta E=E_{\mathrm{pa}}-E_{\mathrm{pc}}\right]$ has been found to increase proportionally, with respect to the scan rate plot (Table 3), (Fig. 7B) revealing the facile electron transfer of the electrolyte and follows eqn (1)-(3).

$$
\begin{aligned}
& I_{\mathrm{c}}(\mathrm{BSA} / \mathrm{anti}-\mathrm{sIL} 2 \mathrm{R} \alpha / \mathrm{CoPS})=18.6 \times 10^{-4} \\
& \times \text { scan rate }\left(\mathrm{mV} \mathrm{s}^{-1}\right)-7.39 \times 10^{-4} \\
& I_{\mathrm{a}}\left(\mathrm{BSA} / \text { anti-sIL2R } \alpha / \mathrm{CoPS}^{-1}\right)=-24.9 \times 10^{-4} \\
& \times \text { scan rate }\left(\mathrm{mV} \mathrm{s}^{-1}\right)-12.2 \times 10^{4}
\end{aligned}
$$

$$
\begin{aligned}
& \Delta E(\mathrm{~V})(\mathrm{BSA} / \mathrm{anti}-\mathrm{sIL} 2 \mathrm{R} \alpha / \mathrm{CoPS})=0.011 \\
& \times \text { scan rate }\left(\mathrm{mV} \mathrm{s}^{-1}\right)+0.601
\end{aligned}
$$

\begin{tabular}{|c|c|c|c|c|c|c|}
\hline Species & Description & Binding energy $(\mathrm{eV})$ & 5 & 10 & 15 & 20 \\
\hline N2 & Neutral amine nitrogen & 399.5 & 41.02 & 23.25 & 26.79 & 44.13 \\
\hline N3 & Oxidized amine (polarn type structure) & 400.8 & 25.13 & 48.95 & 48.49 & 28.07 \\
\hline N4 & Protonated imine (bipolarn type structure) & 402.2 & 13.41 & 12.94 & 9.32 & 3.42 \\
\hline C1 & Neutral $\mathrm{C}-\mathrm{C}$ or $\mathrm{C}-\mathrm{H}$ bonds & 284.5 & 37.44 & 47.80 & 50.58 & 51.65 \\
\hline C4 & C-N bond with bipolarnic type (N4) & 287.4 & 15.53 & 3.29 & 2.97 & 2.10 \\
\hline
\end{tabular}

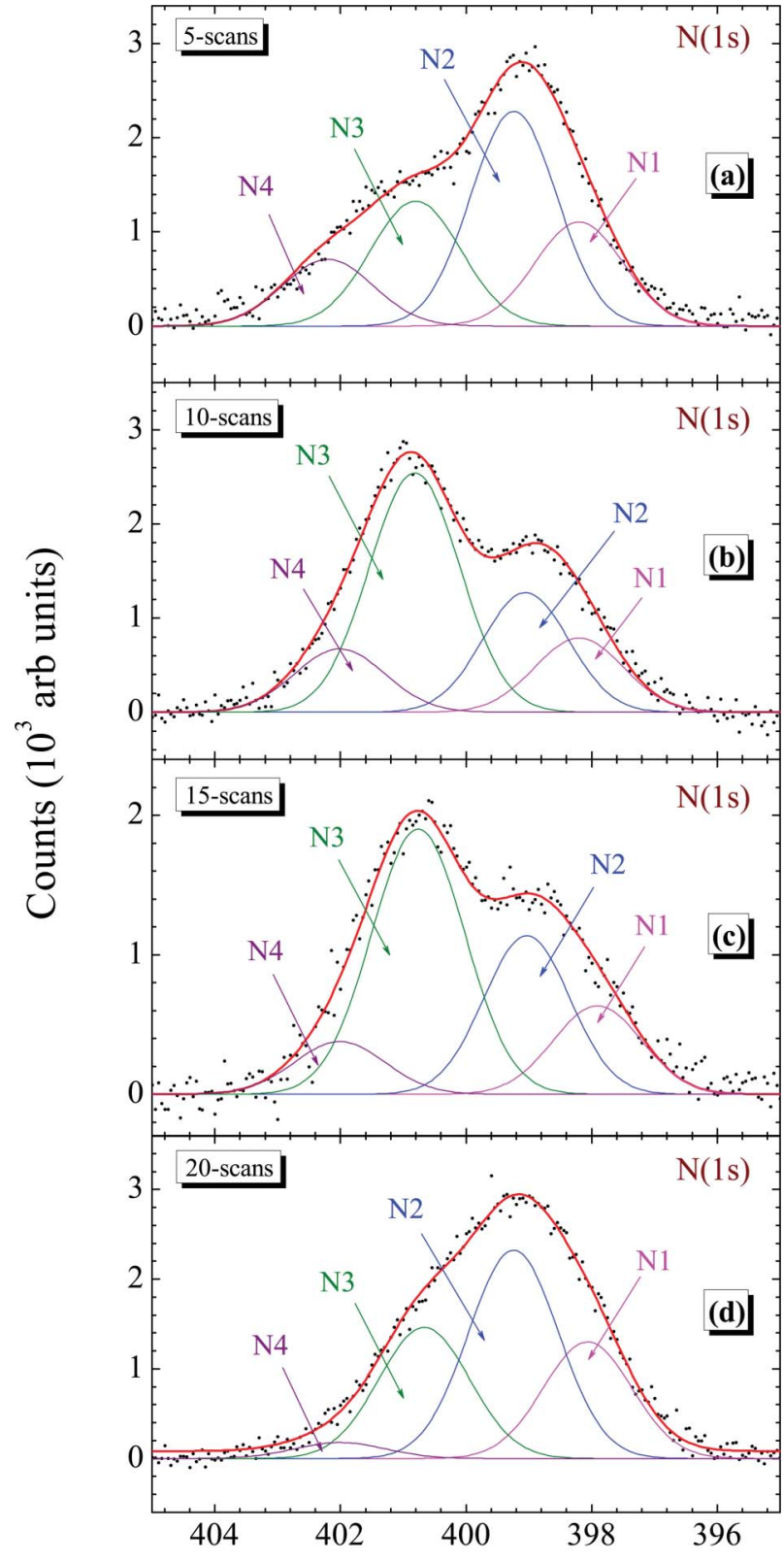

\section{Binding Energy (eV)}

Fig. 4 XPS deconvoluted spectra of nitrogen (1s) as a function of number of polymerization cycles of polyaniline.

Table 2 Detailed description of species obtained from the deconvolution of nitrogen (1s) [Fig. 4] and carbon (1s) [Fig. 5] 


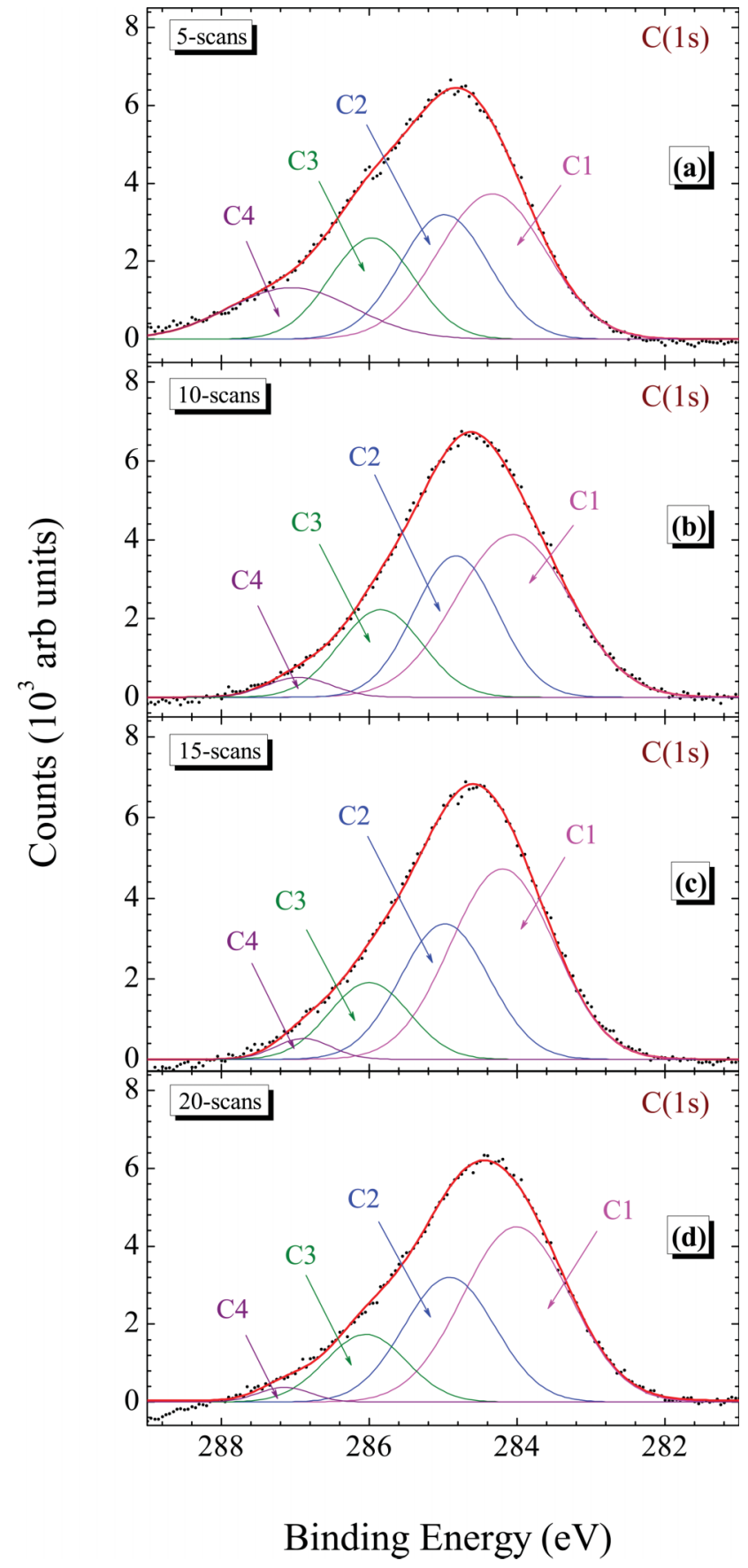

Fig. 5 XPS deconvoluted spectra of carbon (1s) as a function of number of polymerization cycles of polyaniline.

with regression coefficients of $0.995,0.976$ and 0.98 , respectively. The surface concentration $(\tau)$ of the BSA/anti-sIL2R $\alpha /$ Immuno COPS was estimated from the plot of $I_{\mathrm{p}}$ against $v$ from the Brown-Anson model ${ }^{33}$ using the equation:

$$
I_{\mathrm{p}} / v=n^{2} F^{2} A \tau(4 R T)^{-1}
$$

where $n$ represents the number of electrons transferred in the process (1), $F$ is the Faraday constant, $A$ is the surface area of

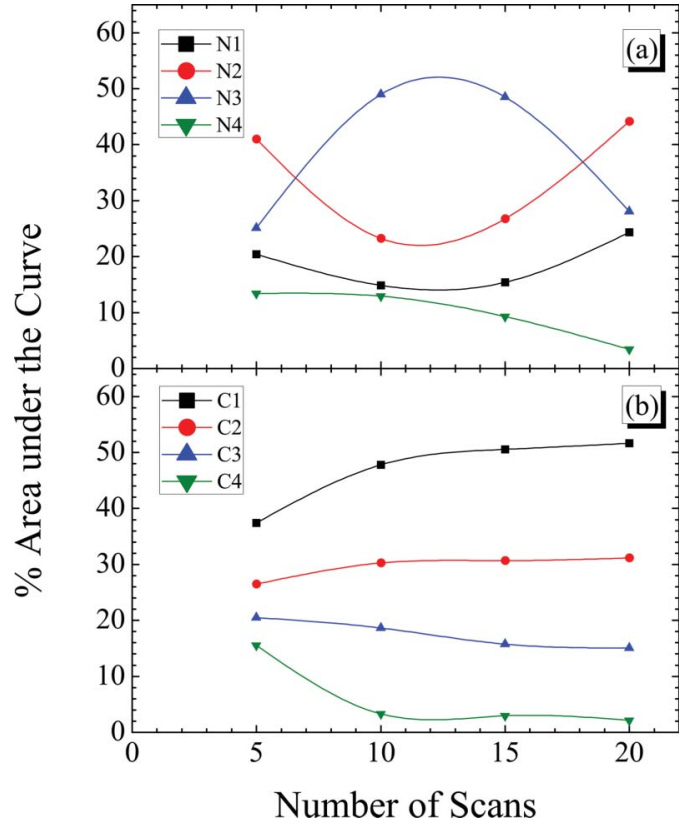

Fig. 6 Variation in the structure of polyaniline as obtained from deconvolution, with respect to the number of scans. (a) Species of nitrogen (1s) from Fig. 4 and (b) species of carbon (1s) from Fig. 5.

the bio-electrode, $\tau$ is the surface concentration of the BSA/ anti-sIL2R $\alpha /$ PPS bio-electrode, $R$ is the gas constant and $T$ is the absolute temperature. The surface concentration was obtained as $6.126 \times 10^{-7} \mathrm{~mol} \mathrm{~cm}^{-2}$.

The electrochemical response studies of the BSA/antisIL2R $\alpha / \mathrm{CoPS}$ immunoelectrode were conducted as a function of the sIL2R $\alpha$ concentration ( 0 to $50 \mathrm{ng} \mathrm{ml}^{-1}$ ) in phosphate buffer (PBS, $50 \mathrm{mM}, \mathrm{pH} 7.2,0.9 \% \mathrm{NaCl}$ ) containing $5 \mathrm{mM}$

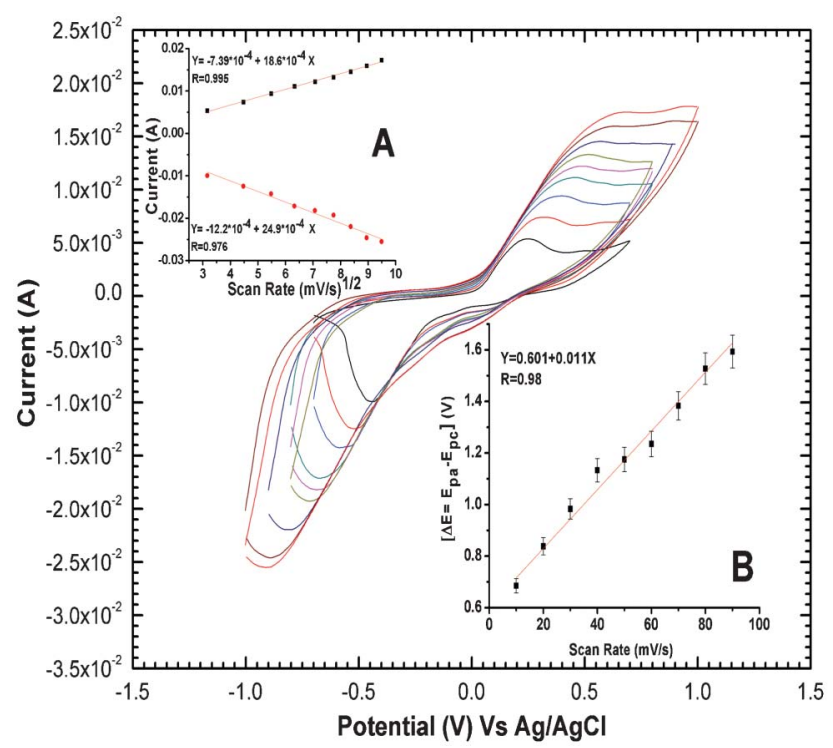

Fig. 7 Cyclic voltammogram (CV) of the Immuno-CoPS bio-electrode as a function of the scan rate. Inset $A$, plot between I (A) and the square root of the scan rate $\left(\mathrm{mV} \mathrm{s}^{-1}\right)$. Inset $\mathrm{B}$, plot between $\Delta E(\mathrm{~V})$ and the scan rate $\left(\mathrm{mV} \mathrm{s}^{-1}\right)$. 
Table 3 Peak to peak separation potential at each scan rate

\begin{tabular}{llllllll}
\hline $\begin{array}{l}\text { Scan rate } \\
\left(\mathrm{mV} \mathrm{s}^{-1}\right)\end{array}$ & $E_{\mathrm{pc}}(\mathrm{V})$ & $E_{\mathrm{pa}}(\mathrm{V})$ & $\Delta E(\mathrm{~V})$ & $\begin{array}{l}\text { Scan } \\
\text { rate }\end{array}$ & $E_{\mathrm{pc}}(\mathrm{V})$ & $E_{\mathrm{pa}}(\mathrm{V})$ & $\Delta E(\mathrm{~V})$ \\
\hline $\mathbf{1 0}$ & -0.438 & 0.247 & 0.685 & $\mathbf{6 0}$ & -0.717 & 0.518 & 1.235 \\
$\mathbf{2 0}$ & -0.520 & 0.318 & 0.838 & $\mathbf{7 0}$ & -0.814 & 0.569 & 1.383 \\
$\mathbf{3 0}$ & -0.585 & 0.398 & 0.983 & $\mathbf{8 0}$ & -0.893 & 0.634 & 1.527 \\
$\mathbf{4 0}$ & -0.675 & 0.458 & 1.133 & $\mathbf{9 0}$ & -0.910 & 0.683 & 1.593 \\
$\mathbf{5 0}$ & -0.688 & 0.487 & 1.175 & & & &
\end{tabular}

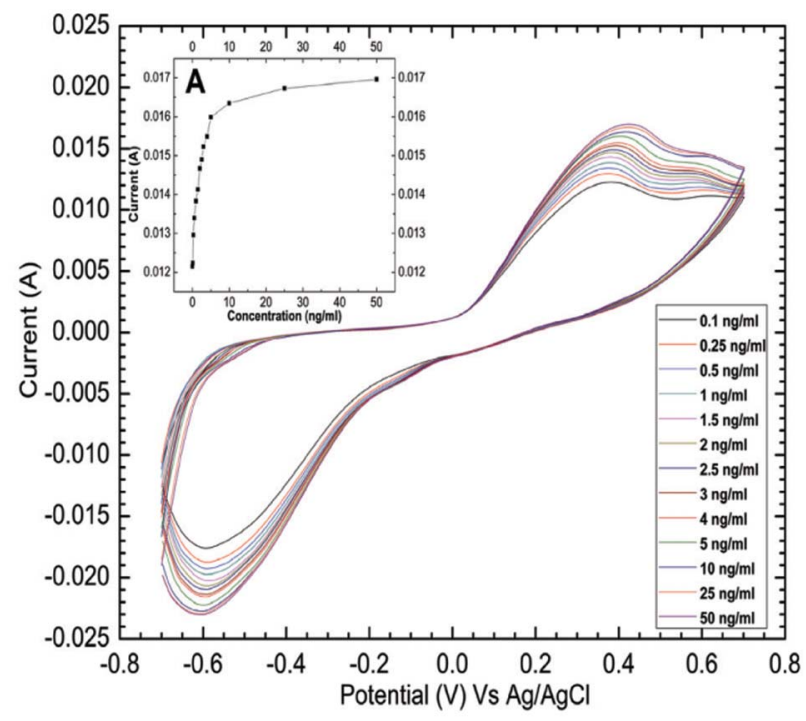

Fig. 8 Electrochemical response studies of the Immuno-CoPS as a function of the soluble interleukin-2 receptor $\alpha$ (sIL2R $\alpha$ ) concentration, at a scan rate of 50 $\mathrm{mV} \mathrm{s}^{-1}$. Inset A, calibration plot between the magnitude of the current and the SIL2R $\alpha$ concentration.

$\left[\mathrm{Fe}(\mathrm{CN})_{6}\right]^{3-/ 4-}$, using cyclic voltammetry (Fig. 8). The magnitude of the current is found to increase on the addition of $\operatorname{sIL} 2 \mathrm{R} \alpha$, due to the formation of an antigen-antibody complex at the electrode surface, between $\operatorname{sIL} 2 \mathrm{R} \alpha$ and anti-sIL2R $\alpha$, that might be due to a favourable conformational change after complex formation that acts as a layer for the faster transfer of electrons. ${ }^{34}$ The reproducibility of the electrode was confirmed by repeating the experiments at least three times. A linear relationship between the magnitude of the current and the SIL2R $\alpha$ concentration is observed (inset Fig. $8 \mathrm{~A}$ ) in the range $0.5-3 \mathrm{ng} \mathrm{ml}^{-1}$ with a sensitivity of $0.737 \mathrm{~mA} \mathrm{ng}^{-1} \mathrm{~mL}^{-1} \mathrm{~cm}^{-2}$, a response time of $150 \mathrm{~s}$ and follows eqn (5):

$$
I(\mathrm{~A})=0.737 \times[\mathrm{sIL} 2 \mathrm{R} \alpha \text { concentration }]+13.07
$$

with a regression coefficient of 0.99 . The association constant $\left(\mathrm{K}_{\mathrm{a}}\right)$ of the antigen-antibody complex formation was calculated using a Hans-Woolf plot and was found to be $3.525 \times 10^{12} \mathrm{~L}$ $\mathrm{mol}^{-1}$, revealing a strong interaction between the $\operatorname{sIL} 2 \mathrm{R} \alpha$ and the anti-sIL2R $\alpha$.

\section{Conclusions}

In conclusion, a novel approach for making conducting paper electrodes for the quantitative estimation of biomarkers has been developed. Conducting paper strips were utilized for the fabrication of Immuno-CoPS, representing a new electrochemical sensing platform for the detection of $\operatorname{sIL} 2 \mathrm{R} \alpha$ in the concentration range from $0.5 \mathrm{ng} \mathrm{ml}^{-1}$ to $3 \mathrm{ng} \mathrm{ml}^{-1}$ with a sensitivity of $0.737 \mathrm{~mA} \mathrm{ng}^{-1} \mathrm{~mL}^{-1} \mathrm{~cm}^{-2}$ and a response time of $150 \mathrm{~s}$. We believe that this approach has great potential for the development of cost effective clinical diagnostics, especially for developing countries.

\section{Acknowledgements}

We thank Professor R. C. Budhani, Director, National Physical Laboratory, India for facilities, encouragement and useful discussions. We thank Dr. A. M. Biradar, NPL and Prof. B. D. Malhotra, Delhi Technological University for encouragement and support. We thank all the members of the Biomedical Instrumentation section, NPL. We sincerely acknowledge Dr. K. N. Sood, NPL (CSIR) for SEM analysis, Mr. M. K. Pandey for technical help and Dr. V. P. S. Awana, NPL (CSIR) for conductivity measurements. The authors gratefully acknowledge financial assistance from the EMPOWER project "Fabrication of intelligent and smart tissue paper based sensor for development of infectious diseases" (CSIR OLP102232) and the Department of Science and Technology Centre on Biomolecular Electronics, India. (DST, India)

\section{References}

1 R. P. Bagwe, L. R. Hilliard and W. Tan, Langmuir, 2006, 22, 4357.

2 S. Santra, P. Zhang, K. Wang, R. Tapec and W. Tan, Anal. Chem., 2001, 73, 4988.

3 J. M. C. S. Magalhaes and A. A. S. C. Machado, Analyst, 2002, 127, 1069.

4 A. Sharma, C. M. Pandey, Z. Matharu, U. Soni, S. Sapra, G. Sumana, M. K. Pandey, T. Chatterjee and B. D. Malhotra, Anal. Chem., 2012, 84, 3082.

5 N. K. Chaki and K. Vijayamohanan, Biosens. Bioelectron., 2002, 17, 1.

6 C. M. Pandey, R. Singh, G. Sumana, M. K. Pandey and B. D. Malhotra, Sens. Actuators, B, 2011, 151, 333.

7 S. K. Sia, V. Linder, B. A. Parviz, A. Siegel and G. M. Whitesides, Angew. Chem., Int. Ed., 2004, 43, 498.

8 Y. Lu, W. Shi, J. Qin and B. Lin, Anal. Chem., 2009, 82, 329.

9 E. Carrilho, S. T. Phillips, S. J. Vella, A. W. Martinez and G. M. Whitesides, Anal. Chem., 2009, 81, 5990.

10 C. M. Cheng, A. W. Martinez, J. Gong, C. R. Mace, S. T. Phillips, E. Carrilho, K. A. Mirica and G. M. Whitesides, Angew. Chem., Int. Ed., 2010, 49, 4771.

11 D. Zang, L. Ge, M. Yan, X. Song and J. Yu, Chem. Commun., 2012, 48, 4683.

12 S. Ge, F. Yu, L. Ge, M. Yan, J. Yu and D. Chen, Analyst, 2012, 137, 4727. 
13 D. Soriano, D. Hugol, N. T. Quang and E. Darai, Fertil. Steril., 2003, 79, 975.

14 T. Ganbo, K.-I. Hisamatsu, H. Inoue, K. Kikushima, A. Mizukoshi and Y. Murakami, Mediators Inflammation, 1995, 4, 350.

15 B. Tan, X. Zhao and C. Yin, Bulletin of Hunan Medical University, 1998, 23, 191.

16 M. Chilosi, G. Pizzolo, G. Semenzato and G. L. Cetto, Int J Biol Markers., 1986, 1(2), 101.

17 R. Kitai, H. Sasaki, K. Matsuda, K. Tsunetoshi, T. Yamauchi, H. Neishi, K. Matsumura, A. Tsunoda, H. Takeuchi, K. Sato and K.-I. Kikuta, Brain Tumor Pathology, 2013, 30(1), 34.

18 R. Malhotra, V. Patel, J. P. Vaque, J. S. Gutkind and J. F. Rusling, Anal. Chem., 2010, 82, 3118.

19 J. Das and S. O. Kelley, Anal. Chem., 2011, 83, 1167.

20 T. Mäkelä, S. Jussila, M. Vilkman, H. Kosonen and R. Korhonen, Synth. Met., 2003, 135-136, 41.

21 J. Huang, I. Ichinose and T. Kunitake, Chem. Commun., 2005, 1717.

22 R. E. Anderson, J. Guan, M. Ricard, G. Dubey, J. Su, G. Lopinski, G. Dorris, O. Bourne and B. Simard, J. Mater. Chem., 2010, 20, 2400.
23 Y. Furukawa, F. Ueda, Y. Hyodo, I. Harada, T. Nakajima and T. Kawagoe, Macromolecules, 1988, 21, 1297.

24 L. Tarachiwin, P. Kiattibutr, L. Ruangchuay, A. Sirivat and J. Schwank, Synth. Met., 2002, 129, 303.

25 S. Yang, H. Yang, H. Ma, S. Guo, F. Cao, J. Gong and Y. Deng, Chem. Commun., 2011, 47, 2619.

26 M. Ibrahim and E. Koglin, Acta Chim Slov., 2005, 52, 159.

27 M. Trchova and J. Stejskal, Pure Appl. Chem., 2011, 83, 1803.

28 C. E. Giacomelli, M. G. E. G. Bremer and W. Norde, J. Colloid Interface Sci., 1999, 220, 13.

29 J. Kong and S. Yu, Acta Biochim. Biophys. Sin., 2007, 39, 549.

30 K. L. Tan, B. T. G. Tan, E. T. Kang and K. G. Neoh, Phys. Rev. B, 1989, 39, 8070.

31 S. N. Kumar, F. Gaillard, G. Bouyssoux and A. Sartre, Synth. Met., 1990, 36, 111.

32 E. T. Kang, K. G. Neoh and K. L. Tan, Prog. Polym. Sci., 1998, 23, 277.

33 P. Kalita, J. Singh, M. K. Singh, P. R. Solanki, G. Sumana and B. D. Malhotra, Appl. Phys. Lett., 2012, 100, 093702.

34 K. K. Jagadeesan, S. Kumar and G. Sumana, Electrochem. Commun., 2012, 20, 71. 